Effect of Sudarshan Kriya Yoga on anxiety, depression, and quality of life in people with type 2 diabetes : A pilot study in Kuwait

\author{
Shiju, Rashmi
}

2019

Shiju , R , Thomas , D , Al Arouj , M , Sharma , P , Tuomilehto , J \& Bennakhi , A 2019 , ' Effect of Sudarshan Kriya Yoga on anxiety, depression, and quality of life in people with type 2 diabetes : A pilot study in Kuwait ', Diabetes \& Metabolic Syndrome: clinical research \& reviews , vol. 13 , no. 3 , pp. 1995-1999 . https://doi.org/10.1016/j.dsx.2019.04.038

http://hdl.handle.net/10138/318041

https://doi.org/10.1016/j.dsx.2019.04.038

publishedVersion

Downloaded from Helda, University of Helsinki institutional repository.

This is an electronic reprint of the original article.

This reprint may differ from the original in pagination and typographic detail.

Please cite the original version. 
Original Article

\title{
Effect of Sudarshan Kriya Yoga on anxiety, depression, and quality of life in people with type 2 diabetes: A pilot study in Kuwait
}

\author{
Rashmi Shiju ${ }^{a}{ }^{*}$, Daisy Thomas a , Monira Al Arouj ${ }^{a}$, Prem Sharma ${ }^{a}$, \\ Jaakko Tuomilehto ${ }^{\mathrm{b}, \mathrm{c}}$, Abdullah Bennakhi ${ }^{\mathrm{a}}$ \\ a Dasman Diabetes Institute, Kuwait \\ ${ }^{\mathrm{b}}$ National Institute of Health and Welfare, Helsinki, Finland

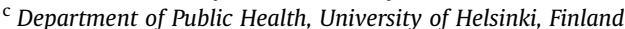

\section{A R T I C L E I N F O}

\section{Article history:}

Received 14 April 2019

Accepted 22 April 2019

\section{Keywords:}

Yoga

Anxiety

Depression

Quality of life

T2DM

SKY

\begin{abstract}
A B S T R A C T
Aim: This pilot study in Kuwait was aimed to assess the effect of Sudarshan kriya yoga (SKY) on anxiety, depression and total quality of life in people with type 2 diabetes mellitus (T2DM).

Methods: 26 T2DM patients aged greater than 30, male and female visiting the outpatient clinic of Dasman Diabetes Institute were enrolled for the study. Pre and post 5 day SKY intervention responses of participants on psychosocial problems were evaluated using four questionnaires (Hamilton anxiety, patient health questionnaire (PHQ-9), Hospital anxiety depression and WHO total quality of life (QOL). Biochemical parameters; such as lipid profile, glycated hemoglobin (HbA1c) were measured at baseline and after 15 weeks of SKY practice.

Results: The mean age of the participants was 56.7 ( $\pm 11.4 \mathrm{SD})$ years, and mean duration of diabetes 15.0 ( $\pm 9.3 \mathrm{SD})$ years. Comparison of responses before and after intervention indicated a significant improvement in the QOL, depression, anxiety and insomnia. But no significant improvement in glycemic control.

Conclusion: Results indicate that SKY can be potentially beneficial for treating anxiety, insomnia, and depression associated in people with T2DM and in improving the quality of life in people with T2DM.
\end{abstract}

(c) 2019 Diabetes India. Published by Elsevier Ltd. All rights reserved.

\section{Background}

Diabetes mellitus is largerly affecting people across the globe rapidly. According to the International Diabetes Federation (IDF) atlas 8th edition, 1 in 11 adults have diabetes and almost 40 million people in the MENA region have diabetes, the figure will rise to 86 million by 2045 if we do not act now [1]. Diabetes is a demanding disease which requires disciplined demeanour. There is enough evidence that depression and anxiety are relatively higher in people with diabetes than in the general population; at least $15 \%$ have clinical depression and it is associated with worse glycemic control and other health problems [2]. Living with diabetes is often a challenge to patients since it disturbs the physical and mental health of the patient. Many seek alternative therapy in addition to conventional medical treatment to manage it. Yoga, a mind-body

\footnotetext{
* Corresponding author. Office of regulatory affairs, Dasman Diabetes Institute, Al-Soor Street, Kuwait, P.O. Box 1180, Dasman, 15462, Kuwait.

E-mail address: rashmi.shiju@dasmaninstitute.org (R. Shiju).
}

therapy originating from India is one of the alternative therapeutic intervention and widely practiced in many countries to maintain the wellness of mind, body and soul. Yoga is considered as a wholesome approach to address the disease condition. Yoga not only cures the physical ailments but also purifies the mind, emotion and whole existence. Teachings of yoga is based on the concept that sickness is not only due to physical condition but result of mental and emotional status. Thus ailments can be eradicated from the root by simultaneous measures taken at physical and mental level. Sage Patanjali, who is called as father of yoga has written yoga sutras thousands of years before in India [3]. Sudarshan Kriya Yoga (SKY) is based on various intricate modulation of breath. It involves repetation of certain pattern of long, short and fast inhalation and exhalation rounds with set duration of holding a breath. Research has demonstrated that regular practice of SKY manifest reduced stress, improved organ function, relaxation and control of psychological and physiological states [4]. A systematic review by Mccall on mechanism of yoga showed a positive impact of yoga on endocrine function, nervous system, physical health, metabolism, circulatory system, behavioral/social, inflammation, psychological 


\section{List of abbreviations}

CAFT Cardiac Autonomic Function test

HADS Hospital Anxiety Depression Scale

HAM-A Hamilton Anxiety Rating scale

PHQ-9 Patient Health Questionnaire

QOL Quality of Life questionnaire

SKY Sudarshan Kriya Yoga

T2DM Type 2 Diabetes Mellitus

status and cognition [5]. Mc Dermott et al. conducted a pilot randomized controlled trial in people with type 2 diabetes (T2DM) for 8 weeks of yoga intervention and concluded that yoga offers a promising lifestyle intervention to decrease body weight and potentially increases psychological well-being [6]. Another randomized trial done in India by P. Jyotsna et al. on 120 people with T2DM to assess the SKY intervention concluded a significant improvement in quality of life (QOL), post-prandial glucose and sympathetic cardiac autonomic function test (CAFT) in people with T2DM practicing SKY [7]. A pre and post study by Kosuri et al. showed a significant reduction in anxiety, general well-being among 35 T2DM patients with 40 day yoga practice [8]. The awareness and practice of yoga is in the nascent stage in Kuwait. To the best of our knowledge no study has been done yet to assess the effect of SKY among population of Kuwait which has a high prevalence of diabetes [9]. In this pilot study we were looking at effects of SKY on quality of life (QOL), depression, and anxiety in people with T2DM residing in Kuwait.

\section{Methods}

Recruitment and eligibility: T2DM patients from Dasman Diabetes Institute were screened for eligibility according to the inclusion and exclusion criteria

Inclusion criteria: People with T2DM aged 30 years and above, scored greater than 15 (mild to severe) according to Hamilton Anxiety Questionnaire, diagnosed with T2DM more than 1 year ago, able to perform yoga postures, breathing exercise and understands English language as the course is delivered in English.

Exclusion criteria: People with type 1 diabetes, on antidepressant drugs, bipolar disorder, pregnant, any acute coronary events in past 6 months, on artificial pace maker, any prior spiritual meditation practice or SKY within 6 months.

Study design and ethical statement: In this prospective study, assessment was carried out at baseline and post SKY intervention on eligible participants after obtaining written informed consent form. The study was approved by the Ethical Review Committee of Dasman Diabetes Institute.The study was registered under clinical trials.gov website with number: NCT02661906.

Intervention: SKY mainly involves kriya, which is a repeatation of breath with long, short and fast inhalation and exhalation according to the sound 'So-Hum' ('So'- inhale, 'Hum'- exhale) played through the recorder by the certified yoga teacher. SKY intervention was provided to the enrolled participants for five days at Dasman Diabetes Institute by certified SKY teachers. Due to cultural taboo, male and female batch of yoga were done at separate premises on the same day with 2-4 certified yoga teachers. The necessary requirements were provided to the enrolled participants. A typical SKY course consists of warm up, sun salutation, asanas, pranayama involving three stages: (bhastrika,ujjayi, alternate nostril breathing and Sudarshan kriya), guided meditation and corpse pose. Participants were provided with instruction manual by the certified yoga teachers as a guide to practice SKY at home daily for 20 min on the last day of the course at Dasman Diabetes Institute.

\subsection{Assessments}

Effect of SKY was assessed through responses of participants on the four questionnaires (HAM-A, total QOL, HADS and PHQ-9) at baseline and after 5 days of SKY intervention. The main endpoint was the mean change from pre-to post intervention on the QOL questionnaire and the secondary endpoints were the mean changes from pre-to post intervention in HAM-A and HADS questionnaire.

Participants fasted for $12 \mathrm{~h}$ and visited Dasman Diabetes Institute clinic at baseline and after 15 weeks of SKY practice at home for clinical parameter measurements. (HbA1C, total cholesterol, low density lipoprotein, high density lipoprotein and triglycerides).

\subsection{Statistical analysis}

Data analysis was carried out using the Statistical Package for Social Sciences, (IBM SPSS Statistics 25.0, IBM Corporation, Armonk, NY, USA). Total scores and domain scores for the questionnaire data were calculated by adding the responses of each participant. Categorical variables have been presented as numbers and percentages, and continuous variables as mean and standard deviation (SD). The paired $t$-test was applied to compare scores between pre and post measures, while McNemar-Bowker test was applied to find the association between pre and post categorical variables. The two-tailed probability value 'p' $<0.05$ was considered statistically significant.

\section{Results}

\subsection{Demographic characteristics}

Table 1 summarizes demographic data of the enrolled participants. Mean age of the study sample was $56.7 \pm 11.4$ SD years and the mean duration of the diabetes $15.0 \pm 9.3$ SD years. Equal number of males and females participated in the study.

Quality of life, Depression and anxiety scores at baseline and post SKY intervention.

QOL questionnaire measured 5 domain score comprising general health, symptom botherness, emotional/mental health, diet satisfaction and physical endurance. The total pre and post SKY intervention scores of the participants showed a statistically

Table 1

Demographics and anthropometric characteristics of study participants at baseline $(\mathrm{N}=26)$.

\begin{tabular}{ll}
\hline Characteristic & $\mathrm{N}(\%)$ \\
\hline Gender & $13(50.0)$ \\
Male & $13(50.0)$ \\
Female & \\
Nationality & $13(50.0)$ \\
Kuwaiti & $6(23.0)$ \\
Egyptian & $5(19.0)$ \\
Indian & $2(8.0)$ \\
Iranian/Other & \\
Age in years & $16(61.5)$ \\
$\leq 60$ & $10(38.5)$ \\
$>60$ & $56.7 \pm 11.4(33-78)$ \\
Mean Age \pm SD (range) & $165.4 \pm 9.1(153.0-183.5)$ \\
Mean Height (cm) \pm SD (range) & $83.1 \pm 19.5(56.5-127.0)$ \\
Mean Weight (Kg) \pm SD (range) & $30.4 \pm 7.2(21.8-53.6)$ \\
Mean BMI \pm SD (range) & $100.4 \pm 14.5(59.0-130.0)$ \\
Mean WC (cm) \pm SD (range) & $15.0 \pm 9.3(1-37)$ \\
Duration of Diabetes (year) &
\end{tabular}


significant improvement in the QOL $(\mathrm{p}=0.011)$. Mean differences in the score domains were (Table 2$)$ : general health $0.7(p=0.205)$, symptom botherness $0.2(\mathrm{p}=0.746)$, emotional/mental health 2.5 $(\mathrm{p}=0.001)$, diet satisfaction $0.9(\mathrm{p}=0.045)$ and physical endurance $2.6(\mathrm{p}=0.011)$.

Significant changes were observed for the HAM-A total score ( $\left.p^{<} 0.001\right)$, for the PHQ-9 total score $\left(p^{<} 0.001\right)$ and for the HADS total score ( $\mathrm{p}=0.002$ ) (Fig. 1$)$.

HAM-A questionnaire is a 14- item test which evaluates anxious mood, tension, fears, insomnia and intellectual domain [10]. Anxious mood, tension, depression, somatic muscular, somatic sensory and insomnia reported as "severe/very severe" by the participants before SKY reduced to either "not present" or "moderate" after SKY intervention. Significant improvements were observed in the state of anxiety $(\mathrm{p}=0.002)$, tension $(\mathrm{p}=0.002)$, depression $(p=0.029)$, insomnia $(p=0.005)$, somatic sensory $(\mathrm{p}=0.003)$ and somatic muscular $(\mathrm{p}=0.010)$, while the responses related to intellectual, cardiovascular symptoms, respiratory symptoms and fear did not improve significantly (Table 3).

\subsection{Glycemic control}

Participants were instructed to practice SKY at home daily for 15 weeks. Clinical parameters measured before the intervention and after 15 weeks did not indicate improvement. Low compliance rate to the SKY practice at home might be the reason for insignificant improvements. $90 \%$ of the participants did not practice SKY at home daily as instructed during their visit at Dasman Diabetes Institute for 15 weeks assessment.

\section{Discussion}

Effect of yoga is extensively researched around the globe during 20 th century [11]. However, to the best of our knowledge, studies to evaluate effects associated with specially SKY on the anxiety, QOL and depression among people with T2DM from Gulf countries are not known. Yoga is in a preliminary stage in the State of Kuwait. In this pilot study we attempted to assess the effect of SKY on psychosocial problems in people with T2DM in Kuwait. This study results indicated significant improvements in total quality of life in T2DM participants which is consistent with PJyotsna et al. study [7] where significant improvement observed in the total mean score of QOL among the SKY practicing group in comparison with the control group.

Similarly significant change were noted for the mean anxiety score (HAM-A) post SKY intervention. This is consistent with the study done by Martin Katzman et al. [10] however, the SKY intervention is applied to the patients with genralized anxiety disorder with or without comorbidities while in our study it is applied on T2DM patients. Non-significant changes in somatic anxiety (cardiovascular, respiratory, intellectual) may be attributed to non

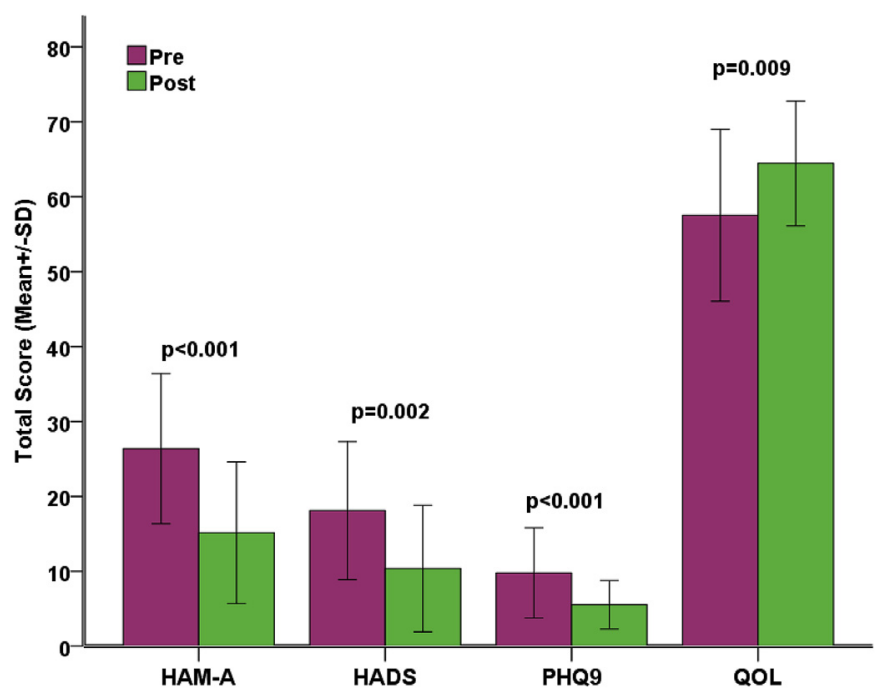

Fig. 1. Pre and Post SKY results on anxiety, depression, quality of life.

affect of SKY on these aspect. Regular practice of yoga has positive impact on hormonal regulations thus reducing anxiety, depression, improving pain, sleep, immune markers [4-6,8,12-14], increase in parasympathetic activity directly influences the nervous system, beneficial effect on cognitive function by reduction in break down and oxygen consumption [15].

Our findings showed a significant decrease in the depression score post SKY intervention.This is consistent with literature $[2,12,16]$ supporting the antidepressant effect of yoga intervention though specifically it is not through SKY intervention.

Participants expressed that they had a very sound and satisfying sleep after a long time with the very first practice of SKY. Out of other symptoms of stress, insomnia is the foremost to respond to the SKY intervention. As SKY treats the cognitive and psychodynamic problems of an individual in relationship with the environment either external or internal $[13,17]$.

In our pilot yoga study, there was no significant improvement in the glycemic control in keeping with the findings of two other studies $[7,18]$ while some other randomized control studies reported significant improvement in glycemic control [19-21]. This variation in findings may be attributed to varying sample size, heterogenous population, type and duration of yoga intervention and compliance to yoga practice.

Plethora of research has been done to prove that chronic stress, anxiety, insomnia, depression and negative mood states are directly and proportionately associated with insulin resistance [22-26], glucose tolerance $[23,27]$, central obesity $[28,29]$, dyslipidemia [30,31], hypertension [31-33] and other components of insulin resistance $[27,34,35]$. Thus there is a dire need of identifying preventive tools to combat the dreadful and epidemic T2DM. Yoga an

Table 2

Quality of life domain scores Domain scores of QOL.

\begin{tabular}{|c|c|c|c|c|c|c|}
\hline & \multirow[t]{3}{*}{ Range of score } & \multirow[t]{3}{*}{$\mathrm{n}$} & \multicolumn{3}{|l|}{ Total score } & \multirow[t]{3}{*}{ p-value } \\
\hline & & & Pre & Post & Difference & \\
\hline & & & Mean \pm SD & Mean \pm SD & Mean & \\
\hline General Health & $3-15$ & 26 & $9.1 \pm 1.9$ & $9.7 \pm 1.9$ & 0.7 & 0.205 \\
\hline Symptom botherness & $3-15$ & 26 & $10.6 \pm 2.1$ & $10.8 \pm 2.1$ & 0.2 & 0.746 \\
\hline Emotional/Mental Health & $3-25$ & 26 & $17.5 \pm 3.8$ & $20.1 \pm 2.7$ & 2.5 & 0.001 \\
\hline Diet satisfaction & $2-10$ & 26 & $7.2 \pm 2.1$ & $8.1 \pm 1.4$ & 0.9 & 0.045 \\
\hline Physical Endurance & $4-20$ & 26 & $13.1 \pm 4.4$ & $15.7 \pm 3.3$ & 2.6 & 0.011 \\
\hline
\end{tabular}

p-values were generated by paired $t$-test. 
Table 3

HAM-A (Anxiety score) PRE AND POST ASSESSMENT.

\begin{tabular}{|c|c|c|c|c|c|c|}
\hline \multirow[t]{3}{*}{ Pre- assessment } & & \multirow[t]{3}{*}{ Total } & \multicolumn{3}{|l|}{ Post } & \multirow{3}{*}{ p-value } \\
\hline & & & Not present/Mild & Moderate & Severe/Very Severe & \\
\hline & & & $\mathrm{n}(\%)$ & $\mathrm{n}(\%)$ & $\mathrm{n}(\%)$ & \\
\hline \multirow[t]{3}{*}{ Anxious mood } & Not present/Mild & 6 & $4(66.7)$ & $1(16.7)$ & $1(16.7)$ & 0.002 \\
\hline & Moderate & 5 & $5(100)$ & $0(0)$ & $0(0)$ & \\
\hline & Severe/Very Severe & 15 & $9(60.0)$ & $6(40)$ & $0(0)$ & \\
\hline \multirow[t]{3}{*}{ Tension } & Not present/Mild & 7 & $7(100)$ & $0(0)$ & $0(0)$ & 0.002 \\
\hline & Moderate & 5 & $3(60.0)$ & $2(40.0)$ & $0(0)$ & \\
\hline & Severe/Very Severe & 14 & $10(71.4)$ & $2(14.3)$ & $2(14.3)$ & \\
\hline \multirow[t]{3}{*}{ Fears } & Not present/Mild & 18 & $15(83.3)$ & $0(0)$ & $3(16.7)$ & 0.072 \\
\hline & Moderate & 6 & $5(83.3) 1$ & $2(16.7)$ & $0(0)$ & \\
\hline & Severe/Very Severe & 2 & $1(50.0)$ & $1(50.0)$ & $0(0)$ & \\
\hline \multirow{3}{*}{ Insomnia } & Not present/Mild & 7 & $6(85.7)$ & $1(14.3)$ & $0(0)$ & 0.005 \\
\hline & Moderate & 9 & $8(88.9)$ & $0(0)$ & $1(11.1)$ & \\
\hline & Severe/Very Severe & 10 & $7(70.0)$ & $2(20.0)$ & $1(10.0)$ & \\
\hline \multirow[t]{6}{*}{ Intellectual } & Not present/Mild & 8 & $5(62.5)$ & $2(25.0)$ & $1(12.5)$ & 0.092 \\
\hline & Moderate & 6 & $2(33.3)$ & $3(50.0)$ & $1(16.7)$ & \\
\hline & Severe/Very Severe & 12 & $8(66.7)$ & $3(25.0)$ & $1(8.3)$ & \\
\hline & & & \multicolumn{4}{|l|}{ Post } \\
\hline & & & Not present/Mild & Moderate & Severe/Very Severe & p-value \\
\hline & & Total & $\mathrm{n}(\%)$ & $\mathrm{n}(\%)$ & $\mathrm{n}(\%)$ & \\
\hline \multirow[t]{3}{*}{ Depressed mood } & Not present/Mild & 11 & $10(90.9)$ & $1(9.1)$ & $0(0)$ & 0.029 \\
\hline & Moderate & 5 & $3(60.0)$ & $2(40.0)$ & $0(0)$ & \\
\hline & Severe/Very Severe & 10 & $6(60.0)$ & $2(20.0)$ & $2(20.0)$ & \\
\hline \multirow[t]{3}{*}{ Somatic/Muscular } & Not present/Mild & 5 & $5(100)$ & $0(0)$ & $0(0)$ & 0.010 \\
\hline & Moderate & 9 & $6(66.7)$ & $1(11.1)$ & $2(22.2)$ & \\
\hline & Severe/Very Severe & 12 & $4(33.3)$ & $5(41.7)$ & $3(25.0)$ & \\
\hline \multirow[t]{3}{*}{ Somatic/sensory } & Not present/Mild & 7 & $6(85.7)$ & $1(14.3)$ & $0(0)$ & 0.003 \\
\hline & Moderate & 6 & $5(83.3)$ & $1(16.7)$ & $0(0)$ & \\
\hline & Severe/Very Severe & 13 & $6(46.2)$ & $5(38.5)$ & $2(15.4)$ & \\
\hline \multirow[t]{3}{*}{ Cardiovascular symptoms } & Not present/Mild & 12 & $10(83.3)$ & $1(8.3)$ & $1(8.3)$ & 0.160 \\
\hline & Moderate & 11 & $5(45.5)$ & $6(54.5)$ & $0(0)$ & \\
\hline & Severe/Very Severe & 3 & $3(100)$ & $0(0)$ & $0(0)$ & \\
\hline \multirow[t]{3}{*}{ Respiratory symptoms } & Not present/Mild & 16 & $13(81.3)$ & $2(12.5)$ & $1(6.3)$ & 0.515 \\
\hline & Moderate & 7 & $5(71.4)$ & $2(28.6)$ & $0(0)$ & \\
\hline & Severe/Very Severe & 3 & $1(33.3)$ & $1(33.3)$ & $1(33.3)$ & \\
\hline
\end{tabular}

alternative therapy might come as a rescue to treat the root cause of the T2DM.

Limitation: Since this was a pilot study several limitation can be listed. The sample size was small and no control group existed. Moreover the data were not blinded. Thus participants might have felt under pressure to score less after the intervention. This might have been ruled out as there were two sets of questionnaire requiring opposite responses post SKY intervention which participants as a layman cannot distinguish. Participants were requested to keep a log of their yoga practice however non compliance was observed. Similar non compliance has been reported in the study by Lana skora-Kondza et al. [18].

\section{Conclusions}

This pilot study done in Kuwait, shows significant improvement in anxiety, depression, QOL and insomnia among the people with T2DM after SKY intervention while glycemic control and lipid profiles did not improve significantly. This may be due to lack of compliance to yoga practice and small sample size. Larger future studies needs to be done to explore effects on glycemic control. SKY may be a potential lifestyle modification for the management of the psychosocial problems associated with T2DM.

\section{Authors' contributions}

RS designed the protocol and wrote the manuscript. DT coordinated participants and collected data. AB and MA screened patients for eligibility and reviewed the manuscript critically. JT reviewed the protocol and manuscript critically. PS performed the statistical analysis and reviewed the manuscript. All authors approved the manuscript.

\section{Acknowledgments}

Authors are indebted to the services rendered by the certified yoga instructors, Mr. Vinod Sharma, Mr. Venkatesh, Mrs Hymavathi Balaji and Mrs. Nandini Sharma. We would also like to acknowledge Art of Living Foundation for providing the support. We are grateful to Kuwait Foundation for the Advancement of Scienes (KFAS) for the financial support. Grant \# RA 2014-028. Last but not the least, we acknowledge all the participants for their wholehearted support.

\section{Appendix A. Supplementary data}

Supplementary data to this article can be found online at https://doi.org/10.1016/j.dsx.2019.04.038.

\section{Disclosure}

The authors declare that they have no competing interests.

\section{References}

[1] International diabetes federation atlas 8 :Middle East and north africa, 
prevalence estimates of diabetes. 2017. http://www.diabetesatlas.org/ resources/2017-atlas.html. [Accessed 20 March 2018].

[2] Lustman PJ, Griffith LS, Freedland KE, Kissel SS, Clouse RE. Cognitive behavior therapy for depression in type 2 diabetes mellitus. A randomized, controlled trial. Ann Intern Med 1998;129:613-21.

[3] Gangadhar BN, Varambally S. Integrating yoga in mental health services. Indian J Med Res 2015;141:747-8.

[4] Brown RP, Gerbarg PL. Sudarshan Kriya Yogic breathing in the treatment of stress, anxiety, and depression. Part II-clinical applications and guidelines. J Altern Complement Med 2005;11:711-7.

[5] McCall MC. How might yoga work? An overview of potential underlying mechanisms. J Yoga Phys Ther 2013;3:1-6.

[6] McDermott KA, Rao MR, Nagarathna R, et al. A yoga intervention for type 2 diabetes risk reduction: a pilot randomized controlled trial. BMC Complement Altern Med 2014;14:212.

[7] Jyotsna VP, Dhawan A, Sreenivas V, Deepak KK, Singla R. Completion report: effect of Comprehensive Yogic Breathing program on type 2 diabetes: a randomized control trial. Indian J Endocrinol Metab 2014;18:582-4.

[8] Kosuri M, Sridhar GR. Yoga practice in diabetes improves physical and psychological outcomes. Metab Syndrome Relat Disord 2009;7:515-7.

[9] Alkandari A, Longenecker JC, Barengo NC, et al. The prevalence of pre-diabetes and diabetes in the Kuwaiti adult population in 2014. Diabetes Res Clin Pract 2018;144:213-23.

[10] Katzman MA, Vermani M, Gerbarg PL, et al. A multicomponent yoga-based, breath intervention program as an adjunctive treatment in patients suffering from generalized anxiety disorder with or without comorbidities. Int J Yoga 2012;5:57-65.

[11] Cramer HLR, Dobos G. Characteristics of randomized controlled trials of yoga: a bibliometric analysis. BMC Complement Altern Med 2014;14:1-20.

[12] Brown RP, Gerbarg PL. Sudarshan Kriya yogic breathing in the treatment of stress, anxiety, and depression: part I-neurophysiologic model. J Altern Complement Med 2005;11:189-201.

[13] Zope SA, Zope RA. Sudarshan kriya yoga: breathing for health. Int J Yoga 2013;6:4-10.

[14] Gupta N, Khera S, Vempati RP, Sharma R, Bijlani RL. Effect of yoga based lifestyle intervention on state and trait anxiety. Indian J Physiol Pharmacol 2006:50:41-7.

[15] Kyizom T, Singh S, Singh KP, Tandon OP, Kumar R. Effect of pranayama \& yoga-asana on cognitive brain functions in type 2 diabetes-P3 event related evoked potential (ERP). Indian J Med Res 2010;131:636-40.

[16] Louie L. The effectiveness of yoga for depression: a critical literature review. Issues Ment Health Nurs 2014;35:265-76.

[17] Agte V, Chiplonkar S. Sudarshan kriya yoga for improving antioxidant status and reducing anxiety in adults. J Altern complementary medicine 2008: $96-100$.

[18] Skoro-Kondza L, Tai SS, Gadelrab R, Drincevic D, Greenhalgh T. Community based yoga classes for type 2 diabetes: an exploratory randomised controlled trial. BMC Health Serv Res 2009;9:33.

[19] Hegde SV, Adhikari P, Kotian S, Pinto VJ, D'Souza S, D'Souza V. Effect of 3 month yoga on oxidative stress in type 2 diabetes with or without complications: a controlled clinical trial. Diabetes Care 2011;34:2208-10.

[20] Beena RK, Sreekumaran E. Yogic practice and diabetes mellitus in geriatric patients. Int J Yoga 2013;6:47-54.

[21] Nagarathna R, Usharani MR, Rao AR, Chaku R, Kulkarni R, Nagendra HR. Efficacy of yoga based life style modification program on medication score and lipid profile in type 2 diabetes - a randomized control study. Int J Diabetes Dev Ctries 2012;32:122-30.

[22] Bjorntorp P. Body fat distribution, insulin resistance, and metabolic diseases. Nutrition 1997:13:795-803.

[23] Vitaliano PP, Scanlan JM, Zhang J, Savage MV, Hirsch IB, Siegler IC. A path model of chronic stress, the metabolic syndrome, and coronary heart disease. Psychosom Med 2002:64:418-35.

[24] Weber B, Schweiger U, Deuschle M, Heuser I. Major depression and impaired glucose tolerance. Exp Clin Endocrinol Diabetes 2000;108:187-90.

[25] Okamura F, Tashiro A, Utumi A, et al. Insulin resistance in patients with depression and its changes during the clinical course of depression: minimal model analysis. Metabolism 2000;49:1255-60.

[26] Raikkonen KM, Kuller LH. The relationship between psychological risk attributes and the metabolic syndrome in healthy women: antecedent or consequence? Metabolism 2002;51:1573-7.

[27] Rosmond R. Stress induced disturbances of the HPA axis: a pathway to Type 2 diabetes? Med Sci Mon Int Med J Exp Clin Res 2003;9. RA35-R39.

[28] Drapeau VTF, Richard D, Tremblay A. Is visceral obesity a physiological adaptation to stress? Panminerva Med 2003;45:7.

[29] Goodman E, Whitaker RC. A prospective study of the role of depression in the development and persistence of adolescent obesity. Pediatrics 2002;110: 497-504.

[30] Chen CCLF, Wu JS, Chang CJ. Correlation between serum lipid concentrations and psychological distress. Psychiatr Res 2001;102:153-62.

[31] Lucini D, Norbiato G, Clerici M, Pagani M. Hemodynamic and autonomic adjustments to real life stress conditions in humans. Hypertension 2002;39: 184-8.

[32] Levenstein S, Smith MW, Kaplan GA. Psychosocial predictors of hypertension in men and women. Arch Intern Med 2001;161:1341-6.

[33] Davidson KJB, Dixon KE, Markovitz JH. Do depression symptoms predict early hypertension incidence in young adults in the CARDIA study? Coronary Artery Risk Development in Young Adults. Arch Intern Med 2000;160:1495-500.

[34] Lustman PJCR. Depression in diabetic patients: the relationship between mood and glycemic control. J Diabet Complicat 2005;19:113-22.

[35] Musselman DL, Betan E, Larsen H, Phillips LS. Relationship of depression to diabetes types 1 and 2: epidemiology, biology, and treatment. Biol Psychiatry 2003; $54: 317-29$. 